\title{
A new integration of hot pressing and carbon partition process to produce high strength steel components with better toughness
}

\author{
Shi-hong Zhang ${ }^{1, a}$, Hong-wu Song ${ }^{1}$, Fei-bao Zhang ${ }^{2}$, and Wei-jie Liu ${ }^{3}$ \\ ${ }^{1}$ Advanced Metal Forming Group, Institute of Metal Research, CAS, Shenyang, China \\ ${ }^{2}$ School of Aeronautical Manufacturing Engineering, Nanchang Hangkong University, \\ Nanchang, China \\ ${ }^{3}$ RIST, Northeastern University, Shenyang, China
}

\begin{abstract}
A novel one step method for hot pressing and quench \& partition (Q\&P) integration - hot Pressing-dynamic partitioning (HP-DP) process is presented, which can be processed by regular hot pressing equipment and process. The HP-DP formed steel is an upgrade of the existing hot pressed steel especially suitable for making high strength components with superior crashworthiness due to better toughness. Corresponding steel sheet based on conventional 22MnB5 is designed and prepared. After that, the physical simulation experiments for HP-DP are carried out on thermal-mechanical simulator. Microstructure of the steel subjected to HP-DP treatment, with a typical Q\&P treated feature, is mainly composed of initial quenched martensite phase, final quenched martensite phase and retained austenite phase, which indicate the occurrence of carbon diffusion concomitantly with martensite transformation. Compared with conventional hot pressed samples, the HP-DP samples show both better tensile property especially elongation and impact energy absorption ability. The effect of HP-DP parameters on the stability of retained austenite and mechanical property is also discussed. The paper illustrates the promising application potential of the HP-DP process.
\end{abstract}

\section{Introduction}

The rapidly increased vehicle population brings many problems such as atmospheric pollution, energy crisis and safety requirement in case of accidents in recent years. This indicates that the automotive industry has to find a solution to improve both the fuel efficiency and the safety of vehicles at the same time. Making use of stronger automotive components is an apparent solution because parts with higher strength can be thinner and lighter, thusly promoting both fuel efficiency and safety [1]. By now, hot pressing is regarded as the only established process to manufacture such automotive parts with strength higher than $1200 \mathrm{MPa}$ [2]. 22MnB5 is commonly used steel grade for this process. The microstructure of the material is ferrite/pearlite at room temperature, and transforms into full martensite after hot pressing. Accordingly, the tensile strength rises from $600 \mathrm{MPa}$ initially to about $1500 \mathrm{MPa}$ [3].

${ }^{\text {a }}$ Corresponding author: shzhang@imr.ac.cn

This is an Open Access article distributed under the terms of the Creative Commons Attribution License 4.0, which permits unrestricted use, distribution, and reproduction in any medium, provided the original work is properly cited. 


\section{MATEC Web of Conferences}

Table 1. Example for composition (wt\%) and Ms temperature for developed Q\& DCP steels.

\begin{tabular}{cccccccccc}
\hline $\mathrm{C}$ & $\mathrm{Si}$ & $\mathrm{Mn}$ & $\mathrm{Ni}+\mathrm{Cr}+\mathrm{Mo}$ & $\mathrm{Cu}$ & $\mathrm{Mo}$ & $\mathrm{B}$ & $\mathrm{Ti}$ & $\mathrm{Al}$ & $\mathrm{M}_{s}(\mathrm{~K})$ \\
\hline 0.19 & 1.55 & 1.53 & 2.47 & 1.01 & 0.0027 & 0.033 & 0.025 & 0.0030 & 583 \\
\hline
\end{tabular}

However, Currently, the elongation of hot-pressed steel, e.g. 22MnB5, is around 6\% [4], which means a relatively low level of product of strength and elongation (PSE)-only around $9 \mathrm{GPa} \%$. Therefore such ultrahigh strength allows a reduction in sheet thickness, but does not have a direct contribution to an increase of crash safety or absorbed crash energy which is determined by the PSE level of the formed part [5]. So the hot-pressed steel becomes insufficient for making vehicle components with good crashworthy qualities.

To further enhance the PSE level of steel, Quenching and partitioning (Q\&P) steel which is first proposed by Speer [6] has been developed over the years. The microstructure of the material, which is composed of a martensitic matrix providing ultrahigh strength and carbon-enriched retained austenite providing good elongation up to $15 \%$, can achieve a PSE level as high as $20 \mathrm{GPa} \bullet \%-30 \mathrm{GPa} \%$ [7]. However, when the temperature-time profile of the Q\&P process is illustrated together with that of the hot pressing process [8], it can be seen that the two profiles are totally incompatible. And the work of Liu et al. [9] further indicated that neither the current hot pressing steel nor the existing Q\&P steel can provide a PSE level higher than $20 \mathrm{GPa} \%$ under the standard hot pressing process.

In this paper, a new one step method for hot pressing and Q\&P integration - hot Pressing-dynamic partitioning (HP-DP) process is proposed, which can be processed by regular hot pressing equipment and process. The developed steel, process design concept and the corresponding microstructure and tensile property are briefly introduced and discussed for the new developed process to show its promising application potential to make high strength automobile components with superior crashworthiness.

\section{Experimental procedures}

\subsection{Design concept of HF-DP process}

Although martensite transformation is often referred to as a diffusionless transformation, the possibility of carbon diffusion from freshly formed martensite to its surrounding untransformed austenite during martensite transformation has been discussed both experimentally [10-12] and theoretically [13]. First of all, the driving force of carbon diffusion is its chemical potential difference in martensite and austenite and thusly is built up as soon as martensite transformation begins. Secondly, the carbon diffusion rate is quite fast in typical temperature range for martensite transformation-it may take from only $7.25 \times 10^{-3}$ to $3.0 \times 10^{-4}$ second to enrich the untransformed austenite [13]. Thus, it is possible that the diffusion of carbon occurs concomitantly with martensite transformation.

Based on the above analysis, Liu [14] proposed the concept of quenching \& dynamic carbon partition concept (Q\&DCP), and developed Q\&DCP steels which compositions are shown in Table 1 as an example [8]. Then the authors proposed the HP-DP process [15], for which the temperature-time profile can be shown in Fig. 1.

\subsection{Experimental methods}

Thermal-mechanical physical compression test with controlled temperature profile are carried out to physically simulate the proposed HP-DP process using the developed steel on a Gleeble 3800 simulator. The experimental specimen and procedure are shown in Fig. 2, in which different deformation at HP process stage and cooling rates during DP process are pre-set. And using the special shape of specimen, tensile tests can be carried out after simulated HP-DP process treatment. For simulated HP-DP specimens before and after tensile tests, the microstructures are characterized by SEM, TEM and XRD 


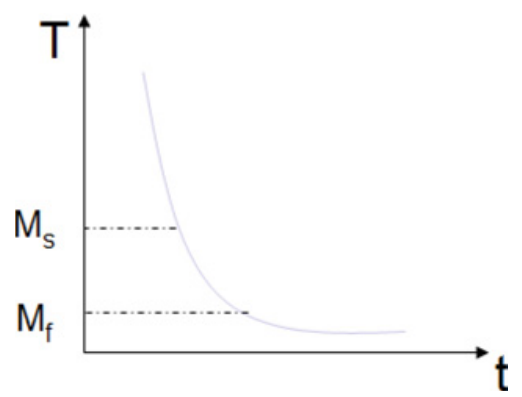

Figure 1. Illustration of temperature-time profile for the proposed HP-DP process.
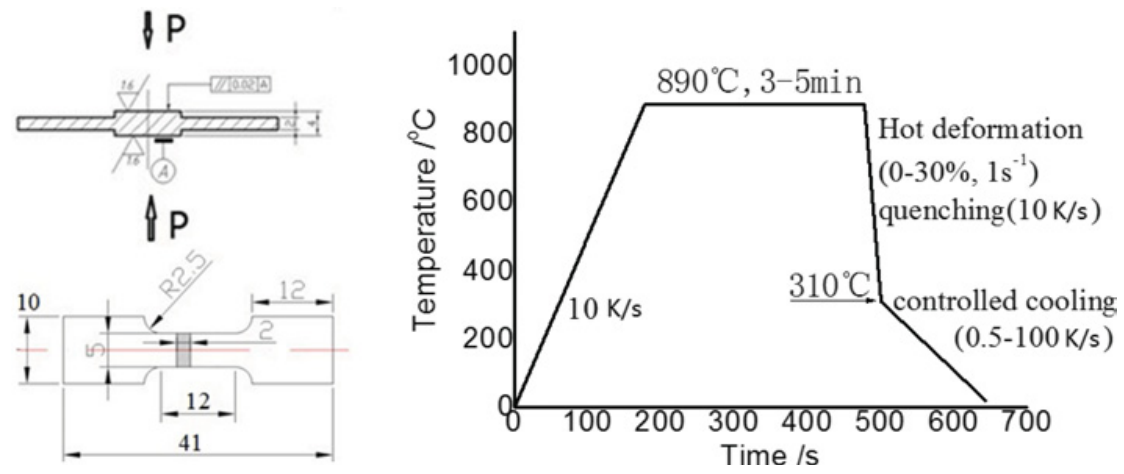

Figure 2. Illustration of the specimen dimension and experimentally simulated HP-DP process.

to investigate the phase constitution and quantitative volume fraction and carbon content of retained austenite.

\section{Results and discussion}

\subsection{Microstructures analysis}

The typical microstructure of HP-DP steel is shown in Fig. 3. Both martensite and retained austenite phases can be found, and further investigation shows both dislocation and twinning martensite phases exists, which verifies carbon diffusion between the martensite and austenite during continuous cooling process.

Quantitative measurements of the volume fraction and carbon content of retained austenite (RA) before and after tensile tests are presented in Fig. 4. It can be obviously seen that the carbon contents of RA are around $1.0 \%$ before tensile tests, much higher than the initial steel content level of 0.19 , which further verified carbon partition and enrichment of RA accrued during HP-DP process. And it also can be seen that the volume fraction of RA reduced significantly after tensile tests, which means martensite transformation and TRIP effect of RA should happen during tensile deformation which will improve the elongation of the steel. Meanwhile, it can be found that the carbon content of RA have obvious increase after tensile tests, which means that the carbon content in the RA in non-uniform and there should be gradient carbon content in the RA as the phase transformation can occur earlier for RA with lower carbon content and achieve increased average carbon content in RA. Thus, multi-martensite phases and retained austenite phase with carbon content gradient are the microstructure characterization of HP-DP steel which is different from both traditional HP steel and Q\&P steel. 


\section{MATEC Web of Conferences}

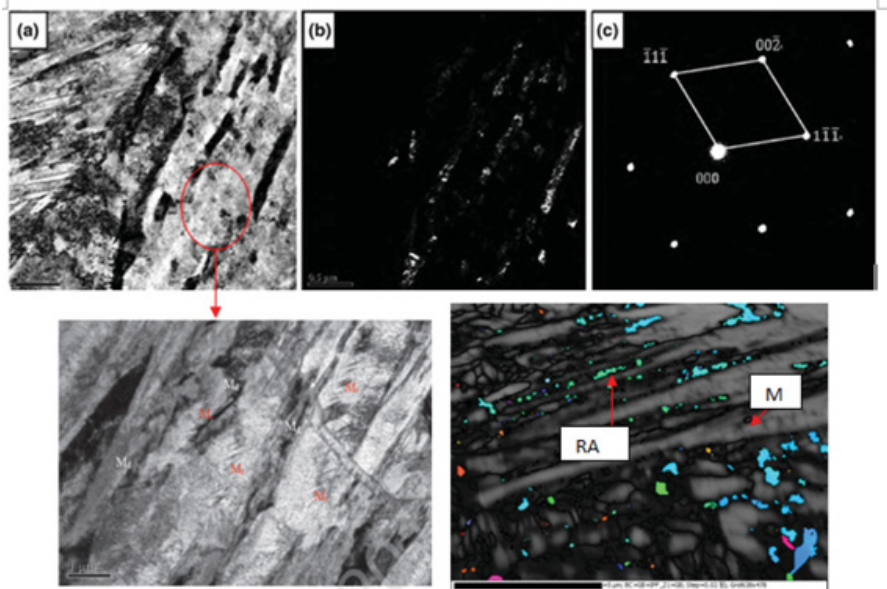

Figure 3. Typical microstructures of HP-DP steel by TEM and EBSD observation.
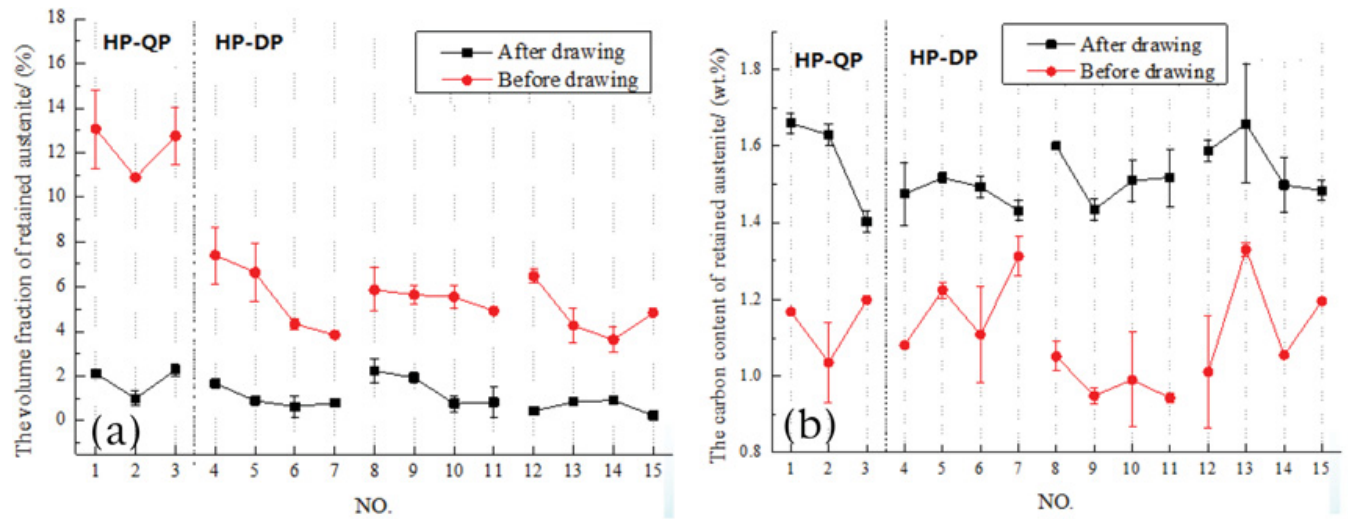

Figure 4. Measured volume fraction and carbon content of RA before and after tensile tests for specimens treated by different HP-DP process parameters (a) volume fraction of RA, (b) average carbon content of RA.

\subsection{Tensile mechanical property}

From Fig. 5, it can be seen that the tensile strengths of HP-DP steels are all in the range of $1500 \mathrm{MPa}-$ $1700 \mathrm{MPa}$, the elongations are mostly in the range of $15.5 \%-16.5 \%$. Both high strength and elongation yields PSE level of HP-DP steel as high as $25 \mathrm{GP} \bullet \%$. And the comparison to the normal HP steel-22MnB5 shows the great enhancement in the PSE level of HP-DP process which should have promising application potential in the future to make high strength automobile components with superior crashworthiness.

\section{Summary}

Based on the concept of quenching-dynamic carbon partition, the integrated hot pressing-dynamic partition process is proposed. Physical simulations of HP-DP process shows significant improvement of PSE level to about two and a half times of the traditional HP process with similar level in tensile strength. The microstructural feature including multi-martensite phases and retained austenite phase with carbon 

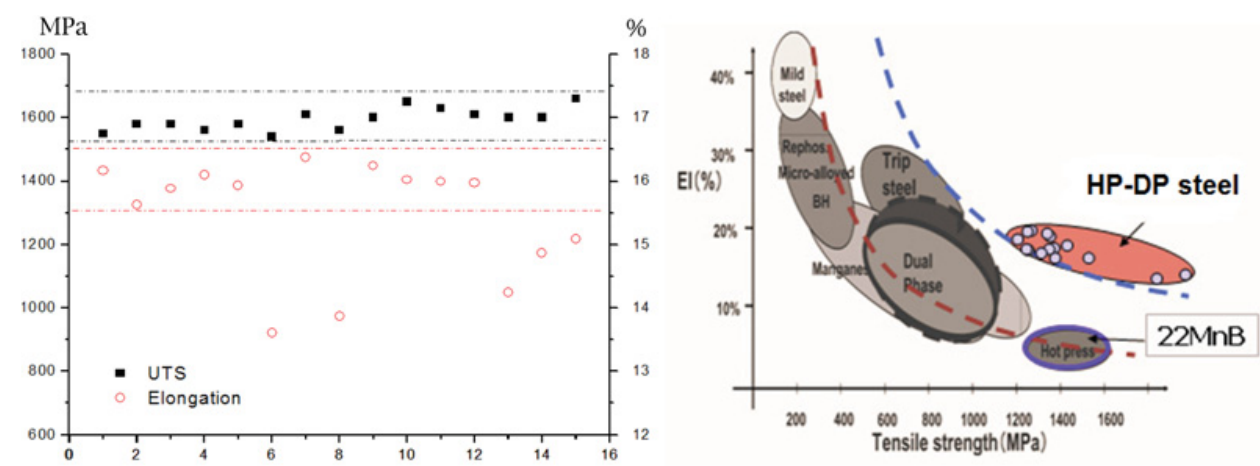

Figure 5. Tensile property for different HP-DP treated steel and comparison with traditional HP steel.

content gradient obtained during HP-DP contributes to the high PSE levels. The fully compatible of HP-DP process with traditional HP process implies the promising application potential of the HP-DP process for making high strength automobile components with superior crashworthiness.

Thanks to the financial support of NSFC project 51034009.

\section{References}

[1] J. Lechler, M. Merklein, In: Materials Science and Technology, Pittsburgh, Pennsylvania (2008)

[2] K.I. Mori, Trans. Nonferrous Met. Soc. China 22, s496 (2012)

[3] H. Karbasian, A.E. Tekkaya, J. Mater. Process. Tech. 210, 5 (2010)

[4] T. Altan, Stamping Journal, 12 (2006)

[5] M. Naderi, Ph.D. Thesis, RWTH Aachen (2007)

[6] J.G. Speer, D.K. Matlock, B.C. De Cooman, Acta Mater. 51, 9 (2003)

[7] D.E. Moor, S. Lacroix, A.J. Clarke, J. Penning, Metall. Mater. Trans. A 39, 11 (2008)

[8] W.J. Liu, Acta Metall. Sin. (Engl. Lett.) 27, 3 (2014)

[9] H. Liu, X. Lu, X. Jin, H. Dong, J. Shi, Scr. Mater. 64, 749 (2011)

[10] B.V.N. Rao, G. Thomas, in International Conference on Martensitic Transformations (HIT Press, Cambridge, 1979)

[11] M. Sarikaya, G. Thomas, J.W. Steeds, in International Conference on Solid-solid Phase Transformations (TMSAIME, Pittsburgh, 1982)

[12] T.Y. Hsu, X. Li, Acta Metall. Sin. 19, 83 (1983)

[13] T.Y. Hsu, J. Phys. IV 5, 351 (1995)

[14] W.J. Liu, a martensite steel based on dynamci carbon partition, Patent, CN 102766818 B

[15] F.B. Zhang, H.W. Song, M. Cheng, X. Li, S.H. Zhang, W.J. Liu, Advanced Materials Research 1063, 42 (2014) 